
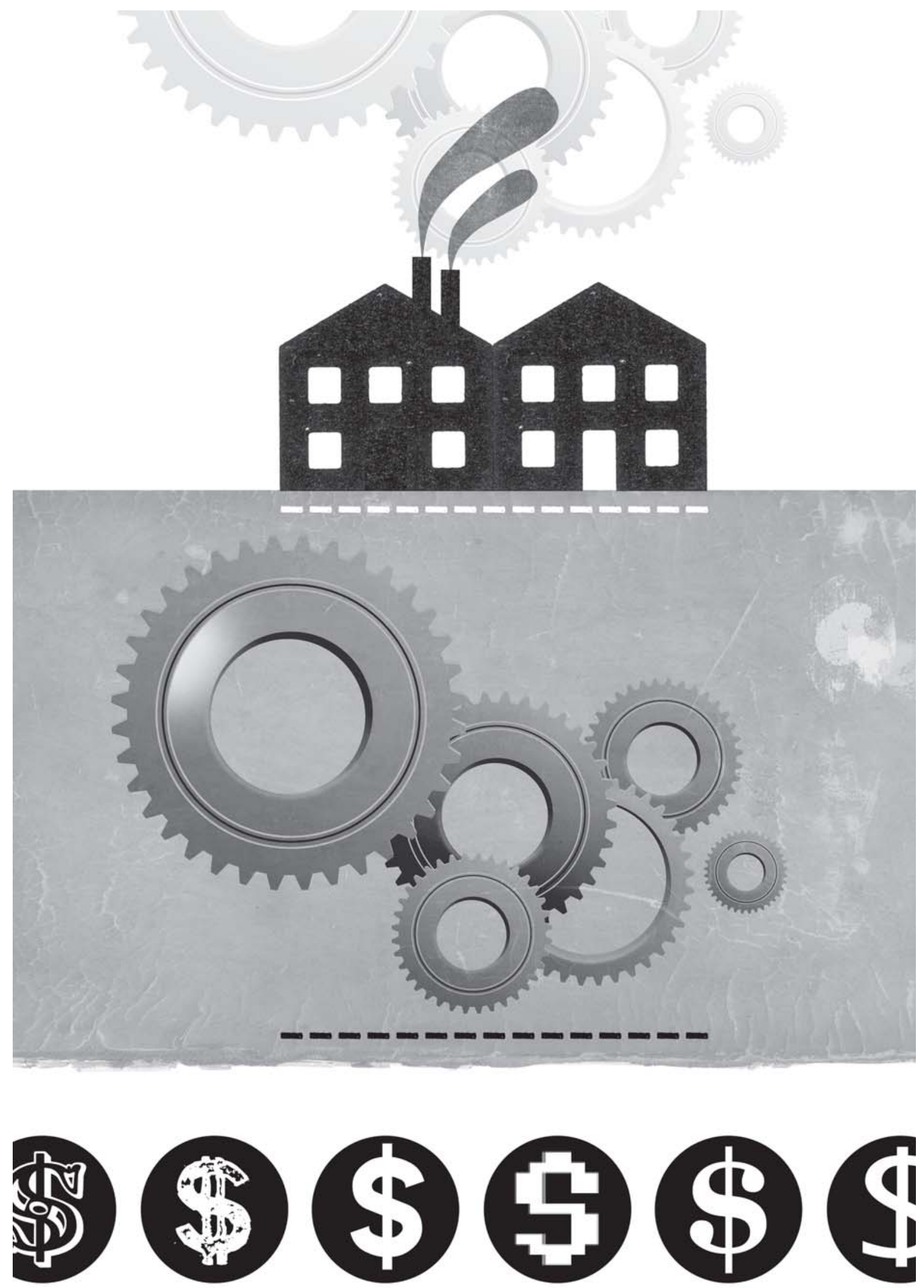


\section{Variedades de Capitalismo e os caminhos da Política Industrial Brasileira - elementos para uma análise comparativa}

\section{Varieties of Capitalism and the paths of the Brazilian Industrial Policy - elements for a comparative analysis}

Ignacio José Godinho Delgado

Professor do PPG em História e do PPG em Ciências Sociais da

Universidade Federal de Juiz de Fora. Pesquisador do Instituto Nacional de

Ciência e Tecnologia - Políticas Públicas e Estratégias de Desenvolvimento (INCT-PPED)

\section{Resumo}

O artigo vale-se da noção de variedades de capitalismo como um recurso heurístico para identificação dos ambientes institucionais que circunscrevem as políticas industriais e o comportamento das empresas em determinados casos nacionais: Brasil, EUA, Alemanha e Coréia do Sul. Ele procura identificar o que há de convergente e os aspectos singulares dos padrões de políticas industriais prevalecentes nestes países desde o final do século passado, apontando sugestões de caráter geral sobre as medidas mais adequadas ao ambiente brasileiro.

Palavras chave: variedades de capitalismo, ambiente institucional, política industrial e de inovação.

\section{Abstract}

The article draws on the notion of varieties of capitalism as a heuristic device to identify the institutional environments that encompass the industrial policies and corporate behavior in specific country cases: Brazil, USA, Germany and South Korea. It seeks to identify what of convergent and unique aspects of industrial policy standards prevailing in these countries since the end of last century, pointing to general suggestions about the measures best suited to the Brazilian environment.

Keywords: varieties of capitalism, institutional environment, industrial and innovation policy 
Ignacio José Godinho Delgado

Neste artigo discorremos inicialmente sobre a noção de variedades de capitalismo e as possibilidades que apresenta para a análise de políticas industriais e de inovação. Destacamos, em seguida, de forma ligeira, o conteúdo e o formato básico das políticas levadas adiante, desde 1998, pelos EUA, Alemanha, Coréia e Brasil, período que, a partir das crises do Brasil e da Coréia, marca o declínio da crença, predominante na década de 1990, segundo a qual os mecanismos de mercado são suficientes para promoção do desenvolvimento. Por fim, assinalamos certos elementos que podem servir à definição de um repertório de políticas mais adequado ao ambiente institucional brasileiro, bem como à fixação de uma agenda de reformas para modificação de aspectos deste ambiente, de modo a desenvolver potencialidades que nele ficam represadas, para maior eficácia das políticas industriais e de inovação ${ }^{1}$.

Políticas industriais buscam, fundamentalmente, criar estímulos ao investimento privado, para objetivos diversos (emparelhamento, inovação, promoção do emprego), através da alteração dos preços relativos e da redução da incerteza quanto ao retorno de tais investimentos. Definida a política, esperase que seja acompanhada de um conjunto articulado, ainda que descentralizado, de decisões de investimento, capazes de provocar efeitos que atendam aos seus objetivos (DELGADO, 2005 e 2009; SUZIGAN \& FURTADO, 2005; CASTRO, 2002; CHANG, 1994). As firmas, contudo, tomam suas decisões em ambientes institucionais diferenciados. A abordagem sobre as variedades de capitalismo fornece as pistas para a identificação dos elementos centrais de tais ambientes. Em sua versão original, ela distingue cinco domínios interativos relevantes para a estratégia empresarial, que tendem a reforçar-se mutuamente (HALL \& SOSKICE, 2001)². A governança corporativa, ao lado dos vínculos entre as empresas e as finanças, é a primeira destas dimensões. Três domínios referemse a diferentes esferas do mundo do trabalho, a saber, questões ligadas à qualificação da mão de obra, às relações industriais e à relação das firmas com seus empregados. Por fim, dilemas de coordenação aparecem também nas relações inter-firmas, associadas aos elos entre fornecedores e clientes ${ }^{3}$.

Hall e Soskice destacaram dois padrões polares de interação estratégica das empresas, num espectro em que formas mistas também podem aparecer. Tais tipos são as economias de mercado liberais, em que prevalecem processos de 
coordenação guiados pelos mecanismos de mercado (os EUA são o paradigma), e as economias de mercado coordenado, em que prevalece a concertação entre os diferentes atores envolvidos (a Alemanha é o paradigma). Em cada um destes casos, desenvolvem-se vantagens institucionais comparativas, que estimulam o apego das empresas às formas de interação predominantes.

No que se refere às atividades de inovação, a abordagem sobre as variedades de capitalismo sugere que as formas liberais favorecem a ocorrência mais acentuada de inovações radicais, ao passo que as formas coordenadas favorecem a ocorrência de inovações incrementais. As primeiras seriam mais afeitas aos fastmoving technology sectors, que envolvem design ligeiro e desenvolvimento rápido de produtos e processos, como a biotecnologia, semicondutores, software, vinculando-se ainda à provisão de componentes para outros setores, como telecomunicações, defesa, dispositivos operacionais do sistema financeiro, defesa e lazer (HALL \& SOKICE, 2001) ${ }^{4}$. A estratégia competitiva das firmas estaria relacionada à capacidade de correr riscos com novos produtos, bem como à implementação rápida. Além dos padrões de financiamento e de governança corporativa, tal estratégia é favorecida pelas relações entre as firmas e o mundo do trabalho, marcadas pelo predomínio do mercado e não da concertação que, embora não conduzam à cooperação para ações inovativas, estimulam a disposição de largar na frente na disputa de mercados, com produtos novos devidamente protegidos pelo segredo industrial, desenvolvidos por laboratórios em geral dissociados e protegidos do ambiente do chão da fábrica e da relação com outros atores, ou, ainda, por novas gerações de empresários. Novidade e preço são os elementos fundamentais da estratégia competitiva.

As inovações incrementais, por seu turno, estariam mais associadas à produção de bens de capital, como máquinas e equipamentos industriais, bens de consumo duráveis, artefatos e equipamentos de transporte (HALL \& SOSKICE, 2001). Neste caso, o que se busca é a introdução de inovações que aperfeiçoem a qualidade de uma linha de produção já estabelecida, de modo a garantir a lealdade do consumidor, além de assegurar a redução progressiva dos custos. A sugestão é que as formas de financiamento e os padrões de governança corporativa das formas coordenadas seriam mais afeitas a este esforço, uma vez que permeáveis às estratégias de longo prazo e à cooperação entre as firmas e outros atores relevantes, como institutos de pesquisa e universidades. Ademais, a relação das firmas com o mundo do trabalho, baseadas na cooperação contínua e na confiança recíproca, predispõem à busca permanente de aperfeiçoamento na produção, embora não favoreçam o segredo industrial. Há pouco espaço para o aparecimento continuado de novas gerações empresariais. Qualidade e lealdade do consumidor são os elementos chaves da estratégia competitiva. 
Ignacio José Godinho Delgado

A abordagem sobre as variedades de capitalismo assinala que as políticas públicas tendem a ser igualmente afetadas pelas características do ambiente institucional (HALL \& SOSKICE, 2001). Assim, nas formas liberais o predomínio do mercado nos processos de coordenação tornaria as empresas mais sensíveis a incentivos propriamente financeiros, ao passo que, nas formas coordenadas a presença de associações vigorosas, capazes de assegurar a lealdade de seus membros para ações concertadas, favorece o desenvolvimento de disposições cooperativas por parte das empresas, sem que o impacto sobre os custos seja tomado como a variável mais importante. No limite, diversos instrumentos de política industrial e tecnológica são usados indiferentemente por todos os países, porém nas formas liberais os incentivos fiscais e subsídios tendem a dispor de maior relevância, além de políticas como as compras governamentais, que permitem a redução da incerteza nos investimentos privados, através de mecanismos de mercado. Nas formas coordenadas é mais provável que prosperem programas de cooperação entre as empresas e destas com o Estado para o alcance de determinadas metas, não obstante a ocorrência de incentivos financeiros imediatos.

Temos lidado com a abordagem sobre as variedades de capitalismo como um instrumento heurístico para identificar, a partir as dimensões interativas apontadas acima, diferentes configurações nacionais, tomadas não como estruturas holísticas, estáticas, mas, tão somente, como ambientes derivados de escolhas efetuadas em condições determinadas, que geram incentivos para o apego dos atores sociais à sua permanência, porém abertos à mudança, notadamente em situações de crise, a partir da orquestração de coalizões, que podem estabelecer novas configurações ${ }^{5}$ (DELGADO, 2009; DELGADO et alli, 2009d; GOUREVITCH, 1986). Ademais, adicionamos à formulação original, o Estado e a inserção das economias nacionais no mercado mundial como domínios interativos de relevo para a operação das firmas. De fato, o Estado é uma instituição decisiva ao enforcement dos elementos que compõem o ambiente em que tal operação se realiza, qualquer que seja a variedade de capitalismo, assim como as políticas desenvolvidas por seus titulares e agências afetam de forma crucial as decisões empresariais (POLANYI, 1980; OFFE \& RONGE, 1984; HANCKÉ, RHODES \& THATCHER, 2007; SCHMIDT, 2006). Além disto, o gasto público, mesmo nas formas liberais, é fundamental na configuração da demanda e da estrutura do mercado. Por seu turno, as firmas operam em ambientes econômicos nacionais mais ou menos abertos, mais ou menos protegidos, além de localizados em posições centrais, periféricas ou semi-periféricas da economia mundial, conforme a presença de atividades econômicas que encerram maior potencial inovativo, o que torna decisivo para as estratégias empresariais a 
inserção internacional das economias nas quais estão inseridas (ARRIGHI, 1997).

Levando em consideração os elementos apontados como importantes para a identificação das diferentes configurações da economia capitalista, nos EUA predomina o mercado de capitais no financiamento das empresas, ao lado de padrões de governança corporativa marcados pelo controle das gerências sobre a gestão, no âmbito de um padrão pulverizado de propriedade acionária (ABELSHAUSER, 2005; GOUREVITCH \& SHINN, 2005; ALDRIGHI, 2003; NOVAES, 2004; RABELO E SILVEIRA, 1999; GUIMARÃES, 2007). Nas relações industriais prevalecem os contratos precários e atomizados e sindicatos, em geral, frágeis. É reduzida a participação das empresas nos esforços de qualificação, que ficam sob responsabilidade dos indivíduos no mercado ou do Estado, na educação genérica formal.. É também reduzida a colaboração entre empresas e seus empregados para esforços de qualificação ou inovação. Esforços de cooperação entre as firmas têm alcance pequeno, prevalecendo mecanismos de coordenação através do mercado (RAMOS, 2008; ROCHA, 2007; ITIRO, 2003; DUFOVSKY \& DULLES, 2004; CLARK et al, 2002). As relações entre empresários e Estado não dispõem, da mesma forma, de caráter concertado, realizando-se, num cenário pluralista, através da prática do lobby, por meio dos quais as empresas buscam posições de força na definição de medidas regulatórias e na negociação de contratos com o Estado, cujos gastos têm papel decisivo na dinamização da economia e mesmo no desempenho das firmas no que se refere à inovação tecnológica (DAHL, 1968; JENKINS-SMITH \& SEBASTIER, 1993; LEHMBRUCH, \& SCHMITTER1982; SCHMITTER \& STREECK, 1999;SANTOS, 2008). Os EUA são a economia hegemônica na ordem mundial, com presença expressiva no comércio global, embora o mercado interno seja o seu mais importante fator de dinamismo (DELGADO et alli, 2009a).

Na Alemanha, apesar de alterações importantes ocorridas nos últimos anos, os bancos são ainda decisivos no financiamento da produção industrial, com participação elevada na propriedade das empresas, que exibem uma estrutura de propriedade concentrada e padrões de governança marcados pela articulação entre diferentes agentes envolvidos no processo produtivo (ABELSHAUSER, 2005; GUIMARÃES, 2006 e 2007; NOVAES, 2004; GOUREVITCH \& SHINN, 2005; SROUR, 2005; GEROLOMANO et al, 2008). As relações industriais na Alemanha reforçam este padrão, com contratos de longa duração, negociados com sindicatos fortes e associações empresariais que desempenham papéis abrangentes, seja na barganha coletiva, seja em esforços de qualificação e inovação tecnológica. A prática da concertação estende-se à relação das empresas com seus funcionários, que participam de forma expressiva da gestão das firmas 
Ignacio José Godinho Delgado

e de iniciativas para qualificação e inovação (EBBINGHAUS \& VISSER, 2000; KUCKULENTZ, 2007). Os elos entre os empresários e Estado, por seu turno, num cenário neo-corporativo, são mediados pelas associações, em múltiplos canais de intermediação. (LEHMBRUCH, \& SCHMITTER1982; SCHMITTER \& STREECK, 1999; GUIMARÃES, 2006 e 2007a). A Alemanha exibe uma economia aberta, com poucas restrições à importação, tecnologicamente dinâmica, embora sejam de pequena expressão os segmentos industriais ligados ao paradigma microeletrônico (DELGADO et alli, 2009a).

Os bancos públicos são decisivos no financiamento da indústria coreana. A estrutura concentrada da propriedade na Coréia do Sul é acompanhada do controle familiar dos grandes conglomerados, num modelo de governança corporativa hierárquico em que é reduzida a participação de acionistas e trabalhadores (CHO E KIM, 2007; NOVAES, 2004; OECD, 2003a). Os contratos de longa duração, que são uma das marcas das relações industriais na Coréia do Sul, não resultam da concertação entre empresas e sindicatos, em geral fracos e atomizados, acarretando um padrão de cooperação entre empresas e seus funcionários assimétrico, mas com forte identificação dos trabalhadores com as empresas. Estas envolvem-se em atividades de qualificação e recrutam funcionários para ações inovativas, mas deixam pouco espaço para a presença dos mesmos na gestão (VERMA et alli, 1995; CHOONG-YOUNG, 1998; KIM, 2005). Relações de cooperação inter-firmas são comuns no âmbito dos chaebols e entre estes e firmas estrangeiras, mas o Estado impõe rígidos limites para que a cooperação resulte em transferência de tecnologia, ao lado do desenvolvimento de capacidade de inovação endógena. A relação do Estado com o empresariado realiza-se via contatos diretos com os chaebols e a federação industrial que controlam, e tem sido marcada pela forte disposição indutiva do primeiro, embora a consolidação dos chaebols tenha acentuado sua autonomia (GRAHAM, 2003; GUIMARÃES, 2007b; HAGGARD, LIM \& KIM, 2003). A Coréia do Sul é uma economia fortemente integrada ao mercado mundial, mas protegida, revelando grande capacidade de inovação tecnológica (DELGADO et alli, 2009a).

No Brasil predominam o autofinanciamento e os bancos públicos, no financiamento das empresas, não obstante a expansão do mercado de capitais. Prevalecem os conglomerados familiares entre as grandes empresas nacionais, mas é expressiva a presença de empresas estrangeiras. A propriedade é concentrada, e na governança corporativa destacam-se o controle familiar e pequena participação de acionistas e funcionários (FONTES FILHO E PICOLIN 2008; OECD, 2003b; LEAL, CAMURI, 2008; NOVAES, 2004; GEROLOMANO et al., 2008). Nas relações industriais o contrato individual predomina, em meio à presença do Estado na regulação de direitos individuais do trabalho (LOBO, 
2006; VIANNA, 2007). O corporativismo sindical, que rege as organizações patronais e de trabalhadores, convive com crescente diversificação da estrutura de representação de interesses (DINIZ e BOSCHI, 1979, 1993; LEOPOLDI, 2000; DELGADO, 1997). São escassas as ações de colaboração entre empresas e empregados para inovação e qualificação, efetuada por agências semi-públicas, que oferecem formação profissional elementar, ao lado da formação geral, garantida pelo Estado. Ao lado das relações hierárquicas, no interior dos conglomerados familiares, iniciativas para a colaboração entre as firmas têm sido estimuladas pelo Estado, como os fóruns de competitividade, envolvendo os segmentos das cadeias produtivas dos setores, além do apoio a clusters e arranjos produtivos locais, mas seu impacto ainda é pequeno. A relação Estado-empresariado no Brasil tem se efetuado por uma multiplicidade de canais, pluralistas e corporativos, sendo que os últimos, esvaziados na década passada, retomaram sua importância. Todavia, eles têm servido pouco à orquestração de ações concertadas (MANCUSO, 2004; DINIZ e BOSCHI, 1993; DELGADO, 2005). Dotado de vigoroso mercado interno, a economia brasileira tem se mantido relativamente fechada, com níveis ainda elevados de proteção, sem superar as deficiências de sua capacidade de inovação tecnológica (DELGADO et alli, 2009a)..

Nos EUA, é notável a ênfase na regulação do direito de propriedade. O Estado participa, ainda, no financiamento da pesquisa em diversas áreas, com destaque para o setor militar e de energia ${ }^{6}$. Todavia, as compras governamentais são o principal instrumento de política industrial. Embora não exclusivo das economias liberais, tal instrumento é de fato, a principal forma liberal de intervencionismo estatal, que se realiza através de mecanismos de mercado. Os indicadores norte-americanos relativos a investimento, crescimento do produto industrial e inovação podem revelar certa perda de fôlego da economia norteamericana. Todavia partem de um patamar extremamente elevado e revelam como os instrumentos de política levados a efeito pelos EUA têm eficácia, no contexto do ordenamento liberal de sua variedade de capitalismo, para manter em alta a atividade inovativa nos EUA. Problemas de outra ordem referem-se, por um lado, à sustentabilidade de tal política, se levarmos em conta os indicadores de endividamento dos EUA. Por outro lado, nada parece indicar que a inclinação para as inovações radicais, inscrita na variedade liberal de capitalismo, possa dotar os EUA de condições competitivas capazes de enfrentar outros concorrentes em dimensões como a qualidade e o aperfeiçoamento contínuo dos produtos, afetos ao predomínio de inovações incrementais, que tendem a se associar às variedades coordenadas de capitalismo. Não poderá, entretanto, a recente intervenção do governo norte-americano na indústria automobilística, acentuando a dimensão consociativa da governança de suas 
Ignacio José Godinho Delgado

empresas, favorecer uma reorientação deste importante setor da economia dos EUA?

Os diferentes e múltiplos programas estabelecidos e desenvolvidos nos últimos anos na Alemanha replicam várias dimensões próprias da tradição consociativa do país, ao buscarem a articulação de institutos de pesquisa, universidades e empresas ${ }^{7}$. A condição de país líder na construção da União Européia impõe desafios a tal tradição, cujo perfil é marcadamente setorial e regional, mas não a dissolveu. Todavia, é cada vez mais evidente o destaque conferido à necessidade de orientar as instituições de ensino e pesquisa para o mercado, através de mudanças nas formas de remuneração, no incentivo ao registro de patentes. É visível também a preocupação com a regulamentação dos direitos de propriedade e com a criação de diferentes mecanismos para apoiar o empreendedorismo tecnológico e as pequenas empresas, potencialmente menos enrijecidas que as grandes firmas alemãs no que se refere à inclinação para modalidades de inovação não incrementais. Não serão estes elementos indicadores da percepção dos limites das vantagens institucionais competitivas que se associam às variedades coordenadas de capitalismo?

A Coréia do Sul é, definitivamente, um caso especial. Dotada de um executivo forte e de organizações empresariais lideradas pelos principais grupos industriais, o país tem revelado uma grande capacidade de estabelecer metas e objetivos em suas políticas, bem como de responder com agilidade aos desafios que as situações de crise lhe impõem, lidando com um repertório variado de política industrial. Não obstante as medidas de desregulamentação econômica vividas nas últimas décadas e das tentativas de conter o poder dos conglomerados, a Coréia mantém sua tradição de formular políticas com metas de longo prazo, envolvendo as empresas nos organismos públicos de formulação e implementação, preservando os mecanismos de contrapartida ${ }^{8}$. O apoio às pequenas empresas não parece empalidecer o protagonismo dos conglomerados que, em articulação com o Estado, participam, para o bem ou para o mal, de um projeto nacional, que tem na elevação da capacidade de inovação de suas empresas um elemento de destaque, sendo permanentemente reiterado, não obstante a volatilidade política do país.

No Brasil, a ênfase conferida à ampliação da capacidade de inovação das empresas como objetivo fundamental das políticas industriais do país aparece com destaque desde o final da década de $1980^{9}$. O país dispõe do mais complexo sistema de ensino e pesquisa da América Latina; forma em escala crescente um número expressivo de doutores; enfrenta dificuldades nos esforços de qualificação da força de trabalho, mas dispõe de um sistema de dimensão nacional para este fim; tem instrumentos diversos de apoio à pesquisa e à inovação, que 
envolvem desde fundos, agências financiadoras, legislação que estimula e favorece a articulação entre as empresas e as universidades, mas o impacto destas condições sobre a elevação da capacidade inovativa das empresas é reduzido. As políticas de inovação iniciaram-se com disposições genéricas que apostavam na criação de um ambiente institucional adequado e no incremento de políticas horizontais voltadas para o aperfeiçoamento da infra-estrutura de inovação como condições, no limite, suficientes para desencadear disposições inovativas das empresas. Desde a instituição dos fundos setoriais e dos fóruns de competitividade, ao final do governo Fernando Henrique Cardoso, a sinalização é diversa. Medidas presentes na PITCE e na Lei do Bem, do governo Lula, elevaram as possibilidades de financiamento e os estímulos fiscais à inovação, embora não fixassem metas e contrapartidas. $\mathrm{O}$ resultado no que se refere ao crescimento econômico, à geração de empregos e a elevação da renda parecem positivos, atendendo a um objetivo central do atual governo que é incorporação de novos contingentes ao mercado de consumo de massa nucleado pelas grandes empresas. No que se refere à elevação da capacidade de inovação das empresas, todavia, os resultados não parecem, ainda, expressivos. A Política de Desenvolvimento Produtivo diversifica a abordagem da política industrial brasileira, dando tratamento diferenciado a setores diversos, e sinaliza para o estabelecimento de metas e contrapartidas. Nos próximos anos veremos se altera de forma substantiva nossa trajetória inovativa.

Se tomarmos apenas os documentos e as disposições expressas pelos governos dos países indicados acima revelam-se diversos elementos de convergência. A elevação da capacidade de inovação das empresas, em especial, aparece como objetivo central das políticas industriais de todos eles, associada ao desenvolvimento de ações para domínio das atividades portadoras de futuro. Outro elemento comum é a ênfase conferida às pequenas empresas e ao empreendedorismo tecnológico. A necessidade de articulação entre o Estado e o empresariado, de reorientação do sistema de ciência e tecnologia para a inovação, e de aproximação das universidades e institutos de pesquisa com a iniciativa privada, são também aspectos comuns às políticas ou às suas intenções. Neste quadro, alterações nos sistemas de remuneração dos pesquisadores universitários, estímulos ao registro de patentes pelas universidades, apoio a formas diversas de parcerias entre universidades, institutos de pesquisa e empresas aparecem recorrentemente nos documentos de governo, de política industrial e/ou de inovação.

Todavia, se as trajetórias dos países analisados indicam várias dimensões convergentes no que se refere aos objetivos e ao repertório de políticas adotadas, elas distinguem-se em relação à importância efetivamente conferida às políticas 
Ignacio José Godinho Delgado

de inovação e às orientadas para o desenvolvimento das atividades portadoras de futuro, bem como parecem revelar que tais políticas dispõem de eficácia diversa conforme o ambiente institucional, tal como compreendido a partir dos elementos indicados pela abordagem sobre as variedades de capitalismo. Por que, não obstante a inclinação generalizada pela aproximação entre universidades e empresas, os resultados são tão diversos, no que toca à elevação da capacidade inovativa destas últimas? Se o ambiente que emoldura esta relação não estimular as firmas a tomarem a inovação como um diferencial competitivo, não bastam a abertura do sistema nacional de ciência e tecnologia ao mundo empresarial e a simples elevação dos requisitos de capital humano e a infra-estrutura de pesquisa e inovação. No limite, a disseminação de padrões de tecnologia industrial básica, medidas de certificação, investimento em capital humano e infra-estrutura tecnológica são condições decisivas ao desenvolvimento de esforços inovativos por parte das empresas, mas não são suficientes. A decisão de inovar envolve a percepção de que é possível contornar o custo de oportunidade envolvido em inversões que exigem a mobilização de ativos intangíveis, de retorno incerto. Sem isto, as empresas vão preferir operar nas linhas de menor resistência, acentuando sua competitividade através de outros mecanismos.

O papel das políticas públicas é, entre outros, induzir a mudanças de comportamento, seja por alterações no ambiente institucional, seja calibrando as políticas para a obtenção de resultados positivos, no que se refere á elevação da capacidade de inovação das empresas, ainda que não alteradas os elementos centrais do ambiente institucional. Neste sentido, qual poderá ser o caminho do Brasil? Em nossa trajetória tiveram relevo a presença de relações precárias no mercado de trabalho, a reduzida cooperação entre as firmas, o predomínio da produção voltada para dentro, as formas mistas de financiamento - mas com peso expressivo do Estado -, o controle familiar da propriedade - mas com elevada presença estrangeira -, em meio à persistência de formas corporativas de intermediação entre Estado e empresariado. Como, e a que custo, tais características podem combinar-se à elevação da capacidade inovativa das empresas brasileiras? O que pode ser reformado ou fortalecido no ambiente institucional? Como elevar a capacidade indutora do Estado e através de quais mecanismos e instrumentos de política industrial e de inovação?

Consideradas as características das relações industriais e dos mecanismos de cooperação entre as firmas, o Brasil aproxima-se das formas liberais de capitalismo, o que torna o mecanismo das compras governamentais um elemento central para as políticas públicas voltadas para a inovação. Neste sentido, deve tornar-se parte central da agenda do governo brasileiro a adaptação da 
legislação a este intento, assegurando que o processo de compras governamentais confira mais importância à qualidade e aos componentes tecnológicos dos produtos do que ao preço. Ademais, deve ser capaz de vir acompanhado de compromissos das empresas com a atividade de inovação. Ao contrário dos EUA, o Brasil não dispõe de um setor de defesa capaz de operar como guardachuva para o desenvolvimento de atividades inovadoras, com potencial irradiador sobre todo o tecido industrial. Todavia, o mapeamento das oportunidades no setor energético, no complexo industrial da saúde, na infraestrutura, dentre outros, permite vislumbrar um cenário de enormes possibilidades de utilização das compras governamentais como fator de estímulo à inovação e ao desenvolvimento de atividades portadoras de futuro, vitais a uma mudança de patamar da economia brasileira na estrutura da economia mundial.

Talvez a estrutura industrial brasileira seja muito complexa e diversificada para permitir mecanismos de indução como os desenvolvidos pela Coréia, em que a combinação de controle estatal do sistema bancário e a presença do sistema de contrapartidas favorecem a inclinação das empresas para atividades inovadoras, especialmente para a exportação. Contudo, o peso que tem o BNDES no financiamento da atividade industrial confere ao poder público brasileiro um poder relativamente pouco utilizado. O desafio é acentuar as exigências de contrapartidas em ações inovativas na concessão de financiamento, associadas a medidas que assegurem aos empresários confiança para a realização de tais inversões, no âmbito da segurança de crédito e do segredo industrial. Se a produção para a exportação é tendencialmente mais apta a beneficiar-se deste tipo de política, também a produção para o mercado interno pode dela valer-se, dentro da perspectiva de incorporação de amplos contingentes ao mercado de massas nucleado pelas grandes empresas, que, ao lado da elevação da renda, deve envolver a ampliação da capacidade das empresas produzirem mais e mais barato. Adicionalmente, a política de apoio a fusões e aquisições, orientada para a criação de empresas brasileiras capazes de atuar como players internacionais, deveria buscar atingir os setores de atividade tecnologicamente mais dinâmicos, de modo a reduzir o impacto negativo que a elevada internacionalização do mercado interno brasileiro acarreta para as atividades de inovação.

É possível, por outro lado, reforçar os elementos de coordenação que existem no ambiente institucional brasileiro, freqüentemente mitigados pela fragmentação dos interesses empresariais e pelo reduzido poder de sanção de que dispõem determinados organismos. A presença da estrutura corporativa de representação de interesses e a experiência de intermediação neo-corporativa entre Estado e 
Ignacio José Godinho Delgado

empresariado, que se reitera de formas diversas ao longo da trajetória brasileira, está longe de desenvolver todas as suas potencialidades para a orquestração de ações cooperativas para atividades de inovação. A ampliação das atribuições das entidades corporativas pode favorecer este intento, tornando-as mais representativas para assegurar a lealdade de seus membros a programas abrangentes. Por seu turno, experiências como as dos fóruns de competitividade podem ser dinamizadas não só para acentuar a cooperação inter-firmas, mas, também, como mecanismo de mobilização do setor empresarial pelo Estado, no sentido da produção de bens de conteúdo tecnológico que atendam à demanda do setor público.

Simplicidade é o que não se pode exigir do caso brasileiro. Não só nosso legado institucional é marcado pela presença de diferentes arranjos em certa medida conflitantes, como a diversidade de nossa estrutura produtiva torna difícil a definição de um conjunto reduzido de metas, comuns a todos os setores industriais. Dispomos, contudo, de experiência na utilização de um vasto repertório de instrumentos, desde as medidas de isenção tributária, passando pelo financiamento público e as compras governamentais. A PDP, neste sentido, parece responder a um desafio inescapável que é a construção de políticas seletivas para TODOS os setores industriais, mobilizando este conjunto variado de instrumentos. Importa, todavia, estabelecer alguma hierarquia neste processo, de modo a não diluir a importância a ser conferida às ações voltadas ao desenvolvimento de atividades portadoras de maior potencial inovativo. Ademais, é significativo definir procedimentos que permitam a fixação de metas no nível das empresas para o alcance dos objetivos perseguidos pelas políticas setoriais. Por fim, é possível definir uma agenda de reformas institucionais que, considerando as características da governança corporativa das empresas e suas relações com as finanças e o mundo do trabalho, favoreçam a acentuação da colaboração de todos os atores envolvidos na atividade produtiva para processos de inovação. 
${ }^{1}$ Este trabalho foi pela primeira vez apresentado na 46th Annual Conference of the Society of Latin American Studies, na University of Bristol em 09/04/2010. Além da participação do INCTPPED, ele ancora-se em pesquisas desenvolvidas com apoio da Fundação de Amparo à Pesquisa do Estado de Minas Gerais (FAPEMIG) e da Agência Brasileira de Desenvolvimento Industrial (ABDI), especialmente, DELGADO, I. G. et alli (2009a) Modelos Econômicos de Capitalismo: análise comparativa dos ambientes institucionais de negócios nos EUA, Alemanha, Coréia do Sul, Espanha, Argentina, México e Brasil; DELGADO, I. G. et alli (2009b) Política Industrial: objetivos e instrumentos; DELGADO, I. G. et alli (2009c) A Política Industrial Brasileira Para Setores Selecionados e a Experiência Internacional, Relatórios de Pesquisa (Produtos 2, 4 e 5) apresentados à Agência Brasileira de Desenvolvimento Industrial (ABDI) para o Projeto Estudo comparativo de política industrial: as trajetórias do Brasil, Argentina, México, Coréia do Sul, EUA, Espanha e Alemanha, coordenado por mim e desenvolvido com a participação de Eduardo Salomão Condé, Helena da Motta Salles e Ângelo Brigato Ésther, também da UFJF. Decisivo para o andamento do projeto foi a participação dos estudantes Breno Vieira de Freitas, Bruna Paiva Lenzi, Carlos Eduardo Ferreira Fernandes, Diogo de Carvalho Antunes Silva, Diogo Gomes de Campos, Fernando Marcus Nascimento Vianini, Heitor de Andrade Carvalho Loureiro, Maedison de Souza, Priscila Musquim Alcântara, Rene Eberle Rocha, William Rezende Alves Ponte.

2 Um balanço das diferentes versões analíticas que focalizam modalidades diversas de capitalismo na ordem econômica contemporânea é efetuada por Jackson e Degg (2006). Não obstante a riqueza das diversas abordagens indicadas por estes autores, entendemos que as formulações de Hall e Soskice oferecem um valioso instrumento heurístico, desde que devidamente retificado, para uma análise abrangente de qualquer país capitalista, a partir da identificação dos elos entre as empresas e diferentes elementos do ambiente no qual operam, no enfrentamento de seus dilemas de coordenação. Ver DELGADO (2009) e DELGADO, ett alli, 2009 d)

${ }^{3}$ A ausência de mecanismos como a hierarquia e a redes pode ser apontado como um dos limites desta tipologia. Ben Ross Schneider incorpora-os na identificação de outros tipos, que entende presentes na América Latina e na Ásia. Não vamos nos estender sobre este ponto, especialmente porque, como ver-se-á adiante, em nosso entendimento, as virtudes analíticas da noção de variedades de capitalismo estão menos associadas à construção de tipos rigidamente articulados por um único mecanismo de interação estratégica - ou princípio alocativo, como sugere Schneider -, mas às suas possibilidades heurísticas, no sentido da identificação e análise dos domínios de interação estratégica das empresas em diferentes países. Conferir SCHNEIDER, 2008

${ }^{4}$ Diferentes dimensões da ordem institucional e do sistema político são tratados como complementaridades institucionais, que se articulam aos domínios interativos apontados acima, reforçando-os. Assim, modelos políticos mais consociativos seriam tendencialmente afeitos às economias coordenadas, ao passo que nas formas liberais predominariam sistemas em que o poder de agenda e de decisão do Executivo é mais acentuado. Do mesmo modo, sistemas de proteção social amplos e reduzidos conectam-se, respectivamente, às formas coordenadas e liberais (HALL \& SOSKICE, 2001).

${ }^{5}$ Conforme Vivien Schmidt, a tentativa de incluir o tema da mudança na teoria das variedades de capitalismo a partir da articulação entre a rational choice, acentuando os interesses dos atores, com o institucionalismo histórico, ressaltando situações de punctuated equilibrium, tal como efetuado por Thelen e Streeck, fracassaria por força do caráter fixo das preferências para a rational choice e da noção de path dependance para o institucionalismo histórico. Sugere então que se dê mais atenção às idéias e aos discursos para análise da mudança no âmbito das variedades de capitalismo. Ver SCHMIDT, 2006 e JACKSON \& DEEG, 2006. Não nos parece que a sugestão de Peter Hall, em texto de 2007, segundo a qual as variedades de capitalismo não podem ser tomadas como um elenco fixo de características institucionais, mas como trajetórias abertas à mudança, altere muito a avaliação que toma a versão original da idéia de variedades de capitalismo como essencialmente impermeável à transformação. Na verdade, o autor assinala como pressões exógenas são absorvidas no âmbito das variedades, levando a mudanças pontuais, sem que a articulação interna de seus elementos efetivamente se modifique (HALL, 2007).

${ }^{6}$ Destacam-se, nos dois governos anteriores a Obama, iniciativas como a Technology for America's Economic Growth, A New Direction to Build Economic Strength, de Clinton, e a American Competitiveness Initiative de Bush. Entre ações setoriais, destacamos a National Nanotechnology Initiative, a Homeland Security Research, and Development, o Project BioShield, a National Aeronautics Research and Development Policy, a networking and Information Technology $R \& D$, a Hydrogen fuellinitiative, a Advanced Energy Initiative e o FutureGen. Conferir OECD (1999). STI Outlook 1999 - Country Responses To Policy Questionnaire. Available in 
Ignacio José Godinho Delgado

$<$ http://www.oecd.org/dataoecd/8/43/2754226.pdf > Accessed on 20/10/2009);OECD (2002) STI Outlook 2002 - Country Response To Policy Questionnaire - USA. Available in <http:// www.oecd.org/dataoecd/60/31/2762731.pdf> . pp. 4. Accessed on 21/10/2009; USA-NIST. (2006) American Competitiveness Initiative Available in <http://www.nist.gov/director/reports/ ACIBooklet.pdf> . Accessed on 20/10/2009; USA (2004) Project BioShild . Available in <http:// www.hhs.gov/aspr/barda/bioshield/index.html>. Accessed on 08 de dezembro de 2009; USANASA.(2004)President Bush Offers New Vision For NASA. Available in http://www.nasa.gov/ missions/solarsystem/bush_vision.html. . Accessed on 08 de dezembro de 2009; USA. (2006) National Aeronautics Research and Development Policy. <http://www.ostp.gov/galleries/ Issues/National\%20Aero\%20Policy\%202006\%20(Glossy).pdf>. Accessed on 08 de dezembro de 2009; USA. NITRD. (2008) Interagency Working Group on High End Computing. Available in $<$ http://www.nitrd.gov/Subcommittee/hec.aspx>. Accessed on 08 de dezembro de 2009; USA. NITRD. (2007) Federal High End Computing Update High. Available in <http://www.nitrd.gov/ Subcommittee/hec/2005_sc_hec_bof.pdf.>. Accessed on 08 de dezembro de 2009; USA. Office of Science and Technology Policy. Executive Office of the President. Hydrogen Fuel Initiative. Available in <http://www.ostp.gov/pdf/1 pger hydrogenfueliniative.pdf $>$. Accessed on 08 de dezembro de 2009; USA. Office of Science and Technology Policy. Executive Office of the President. Advanced Energy Initiative - Research and development in the President's 2009 Budget. Available in <http://www.ostp.gov/galleries/budget09/

AdvancedEnergylnitiative1 pager.pdf>. Accessed on 08 de dezembro de 2009; USA. Advanced Energy Initiative. Available in <http://www.lgprogram.energy.gov/energy_booklet.pdf>. Accessed on 08 de dezembro de 2009; USA. "FutureGen Clean Coal Project". Available in <http://www.fossil.energy.gov/programs/powersystems/futuregen/ >. Accessed on 08 de dezembro de 2009.

7 Programas de destaque na Alemanha, muitos deles desenvolvidos em articulação com a União Européia, em diferentes momentos dos governos de Gerard Schröder e Ângela Merkel, são o Industrielle Gemeinschaftsforschung, o InnoRegio - Innovative Stimuli for Region (InnoRegio - Innovative Impulse für die Region, o Framework Programme Biotechnology - Using and Shaping Opportunities, o National Genome Research Network, o Innovation and Jobs in the Information Society of the $21^{\text {st }}$ Century, o Pact for Research and Innovation, o High-Tech Strategy, programa abrangente, de 2006, que inclui diversos ações setoriais o Higher Education Pact 2020 e o Qualifications Initiative. Conferir GERMANY. Future Technologies for Small and Medium Enterprises (ZUTECH), Available in http://www.aif.de/igf/zutech1.php. Accessed on 11/10/2009; OECD (2002); OECD. STI Outlook 2002 - Country Response to Policy Questionnaire - Germany. Available in: <http://www.oecd.org/dataoecd/60/1/2762626.pdf>. Acesso em: 25/10/2009; OECD (2004); OECD. STI Outlook 2004 - Country Response to Policy Questionnaire - Germany. Available in: <http://www.oecd.org/dataoecd/30/42/34242808.pdf>. Accessed on 25/10/2009 OECD STI Outlook 2006 - Policy Questionnaire - Germany. Available in: <http://www.oecd.org/ dataoecd/5/2/38893058.pdf>. Accessed on 25/11/2009; OECD. (2008). Science and Innovation: Country Notes - Germany. Available in: <http://www.oecd.org/dataoecd/17/40/ 41559102.pdf>. Accessed on 25/10/2009; GERMANY.Federal Ministry of Economics and Technology. Technology Policy. Available in: <http://www.bmwi.de/English/Navigation/ technology-policy.html>. Accessed on 10/10/2009; GERMANY.Federal Ministry of Economics and Technology. Industrial Policy. Available in: <http://www.bmwi.de/English/Navigation/ Economy/industrial-policy.html >. Accessed on 10/10/2009; GERMANY. Annual Economic Report 2008. Available in: <http://www.bmwi.de/English/Redaktion/Pdf/annual-economic-report2008, property=pdf, bereich=bmwi,sprache=en, rwb=true.pdf: $>$. Accessed on 28/10/2009; GERMANY.Federal Ministry of Economics and Technology.An innovation-friendly environment. Available in: <http://www.bmwi.de/English/Navigation/Technology-policy/the-informationsociety.html>. Accessed on out/2009; GERMANYNew Impetus for Innovation and Growth - The Six Billion Euro Programme for Research and Development Federal Ministry of Education and Research. New Impetus for Innovation and Growth - The Six Billion Euro Programme for Research and Development. Available in: <http://www.bmbf.de/en/6075.php>. Accessed on 28/10/2009; GERMANY. Federal Ministry of Economics and Technology. German technology policy:

international activities and priorities. Available in: $<$ http://www.bmwi.de/English/Navigation/ Technology-policy/international-technology.html >. Accessed on 25/10/2009; GERMANY. The German Business Portal. The automotive industry in Germany. Available in: http://www.germanbusiness-portal.info/GBP/Redaktion/en/PDF/automotive-industry-in-germany-invest-ingermany, property=pdf, bereich=gbp, sprache $=e n, r w b=$ true.pdf. Accessed on 03/12/ 2009:GERMANY. BMWI. Annual Economic Report 2008 - staying on course. Available in: http:// www.bmwi.de/English/Redaktion/Pdf/annual-economic-report 2008, property=pdf, bereich= bmwi,sprache=en,rwb=true.pdf. Accessed on 03/12/2009; GERMANY. BMWI. Anual Economic Report 2007. Available in: <http://www.bmwi.de/English/Redaktion/Pdf/annual-economic report-2007, property=pdf, bereich=bmwi, sprache $=e n, r w b=$ true. $p d f>$. Acesso 28/11/2009; GERMANY. BMWI. Annual Economic Report 2008 - staying on course. Available in: http:// www.bmwi.de/English/Redaktion/Pdf/annual-economic-report-2008, property=pdf, bereich= bmwi,sprache=en,rwb=true.pdf. Accessed on 27/11/2009; GERMANY. The Federal Government. 
Materials Innovation for Industry and Society. Available in: <http://www.foerderinfo.bund.de/ en/302.php>. Accessed on 03/12/2009; GERMANY. German Business Portal. The Machinery and Equipment Industry in Germany - Industry Overview. Available in: <http://www.germanbusiness-portal.info/GBP/Redaktion/en/PDF/machinery-and-equipment-industry-invest-ingermany, property $=$ pdf, bereich $=$ gbp, sprache $=e n, r w b=$ true.pdf $>$. Accessed on 03/12/2009; GERMANY, BMWI. German Business Portal. Nanotechnology - Small Parts in a Giant Market. Available in: http://www.german-business-portal.info/GBP/Navigation/en/businesslocation, did=232062.html. Accessed on 02/12/09; GERMANY. BMWI. German Business Portal. Biotechnology - From Start-up to Grown-Up - Biotech "Made in Germany" Comes of Age. Available in:For more information about investing in the medical and industrial biotechnology sectors, click here . http://www.german-business-portal.info/GBP/Navigation/en/businesslocation, did=221230.html. Acesso 12/12/2009.

${ }^{8}$ As principais ações desenvolvidas pelo governo coreano desde 1997 estão consubstanciados nos programas da Lei Especial de Ciência e Tecnologia em Inovação, no Plano para Inovação em Ciência e Tecnologia, no Brain Korea 21" (BK21) e no Fronteira do século XXI em P\&D. De perfi setorial destacamos o Plano Básico U-Coréia, o Desenvolvimento da Indústria de Cuidados em Saúde, a Visão Sobre Energia 2030 o Plano Mestre para Reforma da Defesa. Conferir OECD. Directorate for Science, Technology and Industry: STI Outlook. 2000: National

Policies. Kore.2000. Available in: <http://www.oecd.org/dataoecd/25/48/2109999.pdf>. Accessed on 20/10/2009;OECD. STI Outlook 2002 - Country Response to Policy Questionnaire Korea

Available in <http://www.oecd.org/dataoecd/59/47/2762668.pdf > Accessed on 20/10/2009 UNITED NATIONS. A visão de longo prazo para ciência e tecnologia para 2025. Available in <http://unpan1.un.org/intradoc/groups/public/documents/APCITY/UNPAN008049.pdfs Accessed on 20/11/2009; OECD. Science, Technology and Industry Outlook 2004 - Country Response to Policy Questionnaire - Korea. Available in <http://www.oecd.org/dataoecd/30/60/ 34242958.pdf>; OECD Directorate for Science, Technology and Industry: STI Outlook 2002: National Policies. KOREA Available in: <http://www.oecd.org/dataoecd/59/47/2762668.pdf>. Accessed on 20/10/2009; OECD Directorate for Science, Technology and Industry: STI Outlook 2004: National Policies. Korea Available in: <http://www.oecd.org/dataoecd/5/25/ 38893157.pdf> . Accessed on 20/10/2009; KOREA - Ministry of Knowledge Economy. Pharmaceutical/BT 2008: Overview of Korea's Industries - Pharmaceutical/BT 2008 Invest KOREA Published in October 2008. Disponível a partir de <http://www.mke.go.kr/language/eng/ invest/publications.jsp>. Accessed on 08 de dezembro de 2009; KOREA. Auto Parts 2008: Overview of Korea's Industries - Auto Parts 2008 Invest KOREA, Published in October 2008 Available in http://www.mke.go.kr/language/eng/invest/publications.jsp >. Accessed on $08 \mathrm{de}$ dezembro de 2009; KOREA. Ministry of Knowledge Economy Aerospace 2008: Overview of Korea's Industries - Aerospace 2008 Invest KOREA

Published in October 2008. Disponível a partir de <http://www.mke.go.kr/language/eng/ invest/publications.jsp $>$. Accessed on 08 de dezembro de 2009; KOREA. Information and Communications 2008: Overview of Korea's Industries - Information and Communications 2008 Invest KOREA Published in October 2008 Disponível a partir de <http://www.mke.go.kr/ language/eng/invest/publications.jsp >.Accessed on 08 de dezembro de 2009; KOREA. Ministry for Health, Welfare and Family Affairs. Outline of the heath care industries. Available in <http:// english.mohw.go.kr/front_eng/jc/sjc0102mn.jsp?PAR_MENU_ID=1003\&MENU_ID=100302>. Accessed on 08 de dezembro de 2009; KOREA. Vision 2030' calls for active overseas energy development. Available in < http://www.korea.net/News/News/

NewsView.asp?serial_no=20061130033 >. Accessed on 08 de dezembro de 2009; INTERNATIONAL ENERGY AGENCY. Energy Policies of IEA Countries - The Republic of Korea Available in <http://www.iea.org/textbase/nppdf/free/2006/korea2006.pdf $>$. Accessed on 08 de dezembro de 2009; KOREA. Ministry of National Defense. Defense Plane - Outline. Available in $<$ http://www.mnd.mil.kr/mndEng/DefensePolicy/MasterPlan/overview/index.jsp $>$. Accessed on 08 de dezembro de 2009; KOREA. Ministry of Land, Transport and Maritime Affairs. Ship Investment Company. Available in <http://english.mltm.go.kr/USR/WPGE0201/m 19527/ DTL.jsp >. Accessed on 08 de dezembro de 2009.

${ }^{9}$ No Brasil, sob o governo Fernando Henrique Cardoso, a política industrial é definida no documento Política Industrial, Tecnológica e de Comércio Exterior - Reestruturação e Expansão do Sistema Industrial Brasileiro. Sob Lula, há dois documentos básicos: as Diretrizes de Política Industrial, Tecnológica e de Comércio Exterior, de seu primeiro mandato, e a Política de Desenvolvimento Produtivo, de seu segundo mandato. De perfil setorial, destacam-se o regime automotivo, as ações da Petrobrás, no governo Lula, os diferentes programas da Política Industrial, Tecnológica e de Comércio Exterior e da Política de Desenvolvimento Produtivo, para setores diversos, como o Programa nacional de Nanociência e Nanotecnologia e a Política Nacional de Biotecnologia. BRASIL - MDICE (2004). Diretrizes de Política Industrial, Tecnológica e de Comércio Exterior Available in: <www.desenvolvimento.gov.br/arquivo/sdp/pollndustrial/ MedidasPolndus-livreto.pdf>. Accessed on 07/07/2005; BRASIL - MDICE (2005) 
Ignacio José Godinho Delgado

Acompanhamento da Política Industrial, Tecnológica e de Comércio Exterior. Available in $<w w w$.desenvolvimento.gov.br/arquivo/sdp/pollndustrial/MedidasPolndus-livreto.pdf.> Accessed on 03/06/2005; BRASIL - MICT (1996) Política Industrial, Tecnológica e de Comércio Exterior - Reestruturação e Expansão Competitivas do Sistema Industrial Brasileiro; BRASIL-ABDI (2008) Política de Desenvolvimento Produtivo. Brasília: ABDI. Ver, também, DELGADO, et alli, 2009 b e c.

\section{Referências Bibliográficas}

ABELSHAUSER, W. (2005) The dynamics of German Industry: Germany's path toward the new economy and the American challenge. New York and Oxford: Berghahn Books.Acesso em: 02/06/2009.

ALDRIGHI, Dante Mendes. (JUL/SET 2003). The Mechanisms of Corporate Governance in the United States: An Assessment. RBE. Rio de Janeiro 57(3):469-513.

ARRIGHI, G. (1997). A Ilusão do Desenvolvimento. 2 ed. Petrópolis: Vozes.

CASTRO, A. B. "A Rica fauna da Política Industrial e a sua Nova Fronteira". In: Revista Brasileira de Inovação.2002. Vol. 1. $\mathrm{N}^{\circ} 2$.

CHANG, H. The Political Economy of Industrial Policy. London: Macmillan Press, 1994.

CHO, Dong-Sung; KIM, Jootae. (2007). Outside Directors, Ownership Structure and Firm Profitability in Korea. Disponível em http://www3.interscience.wiley. com/cgi-bin/fulltext/117967270/PDFSTART Acesso em: maio de 2009

CHOONG-YONG, A. (setembro/outubro 2008) Reformas Políticas e Econômicas na Coréia. Tradução de Gilmar Masiero e Juliana Simonishi. Disponível em http://www.asiayargentina.com/usp-07.htm. Acesso em 02/06/2009.

CLARK, Paul, DELANEY, John Thomas \& FROST, Ann Christine. (2002).Collective Bargaining:In the Private Sector. Chicago: Industrial relations Research Association.

DELGADO, I. G. (2005) Empresariado e política industrial no governo Lula. In: PAULA, João Antônio (Org.). Adeus ao Desenvolvimento: a opção do Governo Lula. Belo Horizonte: Autêntica.

DELGADO, I. G., CONDÉ, E. S., ESTHER, A. B., SALLES, H. S Estado, Inserção Internacional e Variedades de Capitalismo: elementos para a análise dos capitalismos reais. Paper apresentado no Seminário Internacional INCT-PPED - Promovendo Respostas à Globalização. Rio de Janeiro. INCTPPED/IE-UFRJ. Disponível em http://www.ideiad.com.br/seminariointernacional/arquivo6.pdf

DELGADO, I. G, CONDÉ, E. S., ESTHER, A. B., SALLES, H. S. (2009a) Modelos Econômicos de Capitalismo: análise comparativa dos ambientes institucionais de negócios nos EUA, Alemanha, Coréia do Sul, Espanha, Argentina, México e Brasil. Relatório Parcial (Produto 2) do Projeto Estudo comparativo de política industrial: as trajetórias do Brasil, Argentina, México, Coréia do Sul, EUA, Espanha e Alemanha. ABDI-FUNDEP. Disponível em <http://www.abdi.com.br/?q=system/files/ Modelos_economicos_de_capitalismo.pdf

DELGADO, I. G, CONDÉ, E. S, ESTHER, A. B., SALLES, H. S. (2009b) Política Industrial: objetivos instrumentos. Relatório Parcial (Produto 4) do Projeto Estudo comparativo de política industrial: as trajetórias do Brasil, Argentina, México, Coréia do Sul, EUA, Espanha e Alemanha. ABDI-FUNDEP.

DELGADO, I. G, CONDÉ, E. S., ESTHER, A. B., SALLES, H. S. (2009b) A Política Industrial Brasileira Para Setores Selecionados e a Experiência Internacional. Relatório Parcial (Produto 5) do Projeto Estudo comparativo de política industrial: as trajetórias do Brasil, Argentina, México, Coréia do Sul, EUA, Espanha e Alemanha. ABDI-FUNDEP

DELGADO, I. G. (1997). A Estratégia de um Revés - Estado e Associações Empresariais em Minas, Juiz de Fora, EDUFJF.

DELGADO, I. G. (2009) Varieties of Capitalism and Industrial Politics: The Brazilian Case in a Comparative Perspective. Paper encaminhado ao 21 st World Congress of Political Science International Political Science Association (IPSA), 2009, Santiago-Chile. Disponível em http:// paperroom.ipsa.org/papers/paper_3135.pdf 
DHAL, Robert. (1968). Pluralist Democracy in the United States : Conflict and Consent. Chicago: Rand McNally \& Company.

DINIZ, E. \& BOSCHI, R. (1979) Autonomia e dependência na representação dos interesses industriais. In: Dados. Nº 22.

DINIZ, E. \& BOSCHI, R. Um Novo Empresariado? Balanço de tendências recentes. In: DINIZ, E. \& BOSCHI, R. (1993). Empresários e Modernização econômica : Brasil Anos 90. Florianópolis: Editora da UFSC/IDACON

DUBOFSKY, Melvin \& DULLES, Foster. (2004). Labor in America: A History. Chicago: Harlan Davidson.

EBBINGHAUS, B. \& VISSER, J. (2000) Trade Unions in Western Europe since 1945. London: Macmillan .

FONTES FILHO, Joaquim Rubens. PICOLIN, Lidice Meireles. (nov./dez. 2008). Governança

GEROLOMANO, Mateus Cecílio et al. (maio-ago 2008). Clusters e redes de cooperação de pequenas e médias empresas: observatório europeu, caso alemão e contribuições ao caso brasileiro. Gest. Prod., São Carlos, v. 15, n. 2, p. 351-365.

GOUREVITCH, P. (1986). Politics in Hard Times. Cornell University Press.

GOUREVITCH, P. e SHINN, J. (2005). Political Power \& Corporate Control: the new global politics of corporate governance. Princeton and Oxford: Princeton University Press.

GRAHAM, E. M. (2003). Reforming korea's, Industrial Conglomerates. Institute for Internacional Economics, Washington.

GUIMARÃES, Alexandre Queiroz (2007b). Estado e economia na Coréia do Sul - do estado desenvolvimentista a crise asiática e a recuperação posterior. Disponível em <http://www.sep.org.br/ artigo/741_28c2f23ba90d0a2f88ea42a4edb640a5.pdf?PHPSESSID=93c69bf512f15aacfd4cac $3 a 9 c>$. Acesso em 27/07/2009

GUIMARÃES, Alexandre Queiroz. (2006), O capitalismo coordenado alemão: do boom do pósguerra à Agenda 2010. Lua Nova. São Paulo, 66: 23-56. Disponível em: http://www.scielo.br/pdf/ In/n66/29083.pdf. Acesso em 23/04/09.

GUIMARÃES, Alexandre Queiroz. (2007a), Modelos de capitalismo e Economia Política Comparada: Instituições, Performance e as Respostas Alemã e Japonesa aos Desafios Recentes. Revista de Ciências Sociais. Rio de Janeiro, Vol. 50, n.1.pp. 159 a 188. Disponível em: http://www.scielo.br/pdf/dados/ v50n1/a06v50n1.pdf. Acesso em 23/04/09.

HAGGARD,S.;LIM,W.; KIM,E. (2003). Economic Crisis and Corporate Restructuring in Korea: Reforming the Chaebol. Cambridge: Cambridge University Press.

HALL, P. \& SOSKICE, D. (2001) Varieties of Capitalism. Oxford University Press.

HALL, P.(September 1, 2007). The Evolution of Capitalism in Europe. For Presentation at the Annual Meeting of the American Political Science Association. Chicago, Illinois.

HANCKÉ, B. RHODES, M. \& THATHCHER, M. (2007) "Introduction: Beyond Varieties of Capitalism". In: HANCKÉ, B. RHODES, M. \& THATHCHER, M. (2007) Beyond Varieties of Capitalism: Conflict, Contradictions, and Complementarities in the European Economy. Oxford University Press.

ITIRO, Andrea. (2003). Flexibilização dos mercados de trabalho: um panorama do debate internacional. Pesquisa \& Debate. São Paulo/Brasil, v. 14, n. 1, p. 60-70.

JACKSON, G. \& DEEG, R. (2006) "How many varieties of capitalism" In: Discussion Paper 06/2 . Max Planck- Institut für Gesellschaftsforschung Köln. Cologne, Germany. (www.mpifg.de)

JACKSON, G. \& DEEG, R. (2006) “How many varieties of capitalism” In: Discussion Paper 06/2 . Max Planck- Institut für Gesellschaftsforschung Köln. Cologne, Germany. (www.mpifg.de)

JENKINS-SMITH, H.\& SEBASTIER, P. "The Advocay Coalition Framework: assessment, revisions anda implications for scholars and practioners. In: JENKINS-SMITH, H.\& SEBASTIER, P (eds) Policy Change and Learning: an Advocacy Coalitions Aproach. Boulder: Westwiew, 1993.

KIM, L. (2005). Da Imitação à Inovação - A Dinâmica do Aprendizado tecnológico na Coréia. Campinas: Editora da UNICAMP. 
Ignacio José Godinho Delgado

KITSCHELT, H., LANGE, P. MARKS, G. \& STEPHENS, J. (1999). Continuity and Change in Contemporary Capitalism. Cambridge University Press.

KUCKULENTZ, Anja. (2007).Studies on Continuing Vocational Training in Germany:An Empirical Assessment. Mannheim: Zew Economic Studies.

LEAL, Maria José e CAMURI, Walter César. (2008). A governança corporativa e os modelos mundialmente praticados. Revista de Ciências Gerenciais, v. XII, n.15.

LEHMBRUCH, G . \& SCHMITTER, P. (1982). Patterns of Corporatist Policy-Making. London: Sage Publications.

LEOPOLDI, M. A. (2000). Política e Interesses. São Paulo: Paz e Terra.

LOBO, V. (2005), Os Elos e os Nós: movimento sindical, (des)mercantilização e os excluídos do mercado formal de trabalho no Brasil. Rio de Janeiro - RJ. Tese de Doutorado. IUPERJ.

MANCUSO, W. P. (2004). "O Lobby da Indústria no Congresso Nacional: Empresariado e Política no Brasil Contemporâneo". Dados - Revista de Ciências Sociais, Rio de Janeiro, v. 47, n. 3, p. 505547, 2004.

NOVAES, Ana. (dezembro/2004). Mercado de Capitais: Lições da Experiência Internacional. Rio de Janeiro.. Disponível em: http://iepecdg.com/DISK\%201/Arquivos/Papers/ LicoesdaExperiencialnternacional-AnaNovaes.pdf. Acesso em: 30/03/09

OFFE, C. \& RONGE, V. (1984). Teses sobre a fundamentação do conceito de Estado Capitalista e sobre a pesquisa política de orientação materialista. In: OFF゙E, C. Problemas Estruturais do Estado capitalista. Rio de Janeiro: Tempo Brasileiro.

POLANYI, K. (1980) A Grande Transformação. Rio de Janeiro: Campus.

RABELO, Flávio. SILVEIRA, José Maria da. (jul. 1999). Estruturas de governança e governança corporativa: avançando na direção da integração entre as dimensões competitivas e financeiras. Texto para Discussão. IE/UNICAMP. n. 77.

RAMOS, Sérgio Motejunas. (2008). A economia política e os contratos coletivos de trabalho nos EUA, México e Brasil: aspectos comparativos. São Paulo. Tese em História. Faculdade de Filosofia, Letras e Ciências Humanas da Universidade de São Paulo. Disponível em http://www.teses.usp.br/teses/ disponiveis/8/8137/tde-11092008-161454/. Acesso em 02/06/2009.

ROCHA, J. C. D. (jan. 2007). Direito do Trabalho nos Estados Unidos: Considerações sobre as Bases do Sistema Norte Americano. Revista Jurídica. Disponível em: http://www.facs.br/revistajuridica/ edicao janeiro2007/convidados/con3.doc. Acesso em: 05/2009.

SANTOS, Luiz Alberto (2008). Regulamentação das Atividades de Lobby e seu Impacto Sobre as Relações Entre Políticos, Burocratas e Grupos de Interesse no Ciclo de Políticas Públicas: Análise Comparativa dos Estados Unidos e Brasil. Disponível em < http://bdtd.bce.unb.br/tedesimplificado/ tde_busca/arquivo.php?codArquivo=4798. Acesso em 28/09/2009.

SCHMITD, V. (2006). Bringing the State Back into The Varieties of Capitalism and Discourse into the Explanation of Change. Panel 7-5 Explaining institutional change in different varieties of capitalism. In: Annual Meetings of the American Political Science Association. Philadelphia.

SCHMITTER, P. \& STREECK, W.(1999) "The Organization of Business Interests: Studying the Associative Action of Business in Advanced Industrial Societies". In : MPIfG - Discussion Papers. $N^{\circ}$ 99/1. Germany: Max-Planck-Institut für Gesellschaftsforschung. Disponível em <www.mpi-fg-koelnmpg.de>Acesso em 10/09/2009.

SCHNEIDER, B. (2008), Comparing Capitalisms: Liberal, Coordinated, Network and Hierarchical Varieties (mimeo).

SROUR, Gabriel. (2005). Práticas diferenciadas de governança corporativa: um estudo sobre a conduta e a performance das firmas brasileiras. Rev. Bras. Econ. [online]. vol.59, n.4, pp. 635-674. ISSN 0034-7140. Acesso em 03/05/09.

SUZIGAN, W. \& FURTADO, J Política Industrial e Desenvolvimento, in, PAULA, J. A. Adeus ao Desenvolvimento: a opção do governo Lula, BH, Autêntica, 2005.

TIRONI, Luís F. \& CRUZ, Bruno de O. Inovação Incremental ou Radical: Há motivos para diferenciar? Uma abordagem com dados da PINTEC. Texto para Discussão Nº 1360. Brasília: IPEA. 
VERMA, A. KOCHAN, T. \& LANSBURY,R. (1995). Employment Relations in the Growning Asian Economies. New York, Routledge.

VIANNA, L. W. (1976). Liberalismo e Sindicato no Brasil. Rio de Janeiro: Paz e Terra.

\section{Documentos de Governo e de Agências Multilaterais}

BRASIL - MDICE (2004). Diretrizes de Política Industrial, Tecnológica e de Comércio Exterior. Disponível em: www.desenvolvimento.gov.br/arquivo/sdp/pollndustrial/MedidasPolndus-livreto.pdf $>$. Acesso em $07 / 07 / 2005$

BRASIL - MDICE (2005) Acompanhamento da Política Industrial, Tecnológica e de Comércio Exterior. Disponível em <www.desenvolvimento.gov.br/arquivo/sdp/pollndustrial/MedidasPolnduslivreto.pdf.> Acesso em 03/06/2005.

BRASIL - MICT (1996) Política Industrial, Tecnológica e de Comércio Exterior - Reestruturação e Expansão Competitivas do Sistema Industrial Brasileiro.

BRASIL-ABDI (2008) Política de Desenvolvimento Produtivo. Brasília: ABDI.Disponível em <http:// www.oecd.org/dataoecd/59/47/2762668.pdf> Acesso em 20/10/2009.

GERMANY Federal Ministry of Education and Research. New Impetus for Innovation and Growth - The Six Billion Euro Programme for Research and Development. Disponível em: <http://www.bmbf.de/en/ 6075.php>. Acesso em 28/10/2009.

GERMANY, BMWI. German Business Portal. Nanotechnology - Small Parts in a Giant Market. Disponível em:

GERMANY. Annual Economic Report 2008. Disponível em: <http://www.bmwi.de/English/Redaktion/ Pdf/annual-economic-report-2008, property=pdf, bereich=bmwi, sprache=en, rwb=true.pdf: $>$. Acesso em 28/10/2009.

GERMANY. BMWI. Annual Economic Report 2008 - staying on course. Disponível em: http:// www.bmwi.de/English/Redaktion/Pdf/annual-economic-report-

2008, property=pdf, bereich=bmwi, sprache=en,rwb=true.pdf. Acesso em 03/12/2009.

GERMANY. BMWI. Annual Economic Report 2008 - staying on course. Disponível em: http:// www.bmwi.de/English/Redaktion/Pdf/annual-economic-report-

2008, property=pdf, bereich=bmwi, sprache=en,rwb=true.pdf. Acesso em 27/11/2009.

GERMANY. BMWI. Anual Economic Report 2007. Disponível em: <http://www.bmwi.de/English/ Redaktion/Pdf/annual-economic-report-

2007, property=pdf, bereich=bmwi, sprache=en,rwb=true.pdf $>$. Acesso 28/11/2009

GERMANY. BMWI. German Business Portal. Biotechnology - From Start-up to Grown-Up - Biotech "Made in Germany" Comes of Age. Disponível em: http://www.german-business-portal.info/GBP/ Navigation/en/business-location,did=221230.html. Acesso 12/12/2009.

GERMANY. Federal Ministry of Economics and Technology. German technology policy: international activities and priorities. Disponível em: <http://www.bmwi.de/English/Navigation/Technology-policy/ international-technology.html>. Acesso em 25/10/2009.

GERMANY. Futuro de Tecnologias para Pequenas e Médias Empresas (ZUTECH), Disponível em http:// www.aif.de/igf/zutech1.php. Acesso em 10/11/2009; OECD (2002).

GERMANY. The Federal Government. Materials Innovation for Industry and Society. Disponível em: <http://www.foerderinfo.bund.de/en/302.php>. Acesso em 03/12/2009; GERMANY. German Business Portal. The Machinery and Equipment Industry in Germany - Industry Overview. Disponível em: <http://www.german-business-portal.info/GBP/Redaktion/en/PDF/machinery-and-equipmentindustry-invest-in-germany, property=pdf, bereich=gbp, sprache=en, rwb=true.pdf $>$. Acesso em 03/ $12 / 2009$.

GERMANY. The German Business Portal. The automotive industry in Germany. Disponível em: http:// www.german-business-portal.info/GBP/Redaktion/en/PDF/automotive-industry-in-germany-invest-ingermany, property $=$ pdf, bereich $=$ gbp, sprache $=$ en, $r w b=$ true.pdf. Acesso em 03/12/2009. 
Ignacio José Godinho Delgado

GERMANY.Federal Ministry of Economics and Technology. Industrial Policy. Disponível em: <http:// www.bmwi.de/English/Navigation/Economy/industrial-policy.html>. Acesso em 10/10/2009.

GERMANY.Federal Ministry of Economics and Technology. Technology Policy. Disponível em: <http:// www.bmwi.de/English/Navigation/technology-policy.html>. Acesso em 10/10/2009.

GERMANY.Federal Ministry of Economics and Technology.An innovation-friendly environment. Disponível em: <http://www.bmwi.de/English/Navigation/Technology-policy/the-informationsociety.html>. Acesso em out/2009. http://www.german-business-portal.info/GBP/Navigation/en/ business-location, did=232062.html. Acesso em 02/12/09.

INTERNATIONAL ENERGY AGENCY. .Energy Policies of IEA Countries - The Republic of Republic of Korea Disponível em <http://www.iea.org/textbase/nppdf/free/2006/Republic of Korea2006.pdf>. Acesso em 08 de dezembro de 2009.

OECD (1999). STI Outlook 1999 - Country Responses To Policy Questionnaire. Disponível em <http:// www.oecd.org/dataoecd/8/43/2754226.pdf > p.5. Acesso em 20/10/2009).

OECD (2000). STI Outlook 2000: National Policies - Germany. Disponível em <http://www.oecd.org/ dataoecd/25/4/2109224.pdf>. Acesso em 25/10/2009.

OECD (2002) STI Outlook 2002 - Country Responose To Policy Questionnaire - USA. Disponível em <http://www.oecd.org/dataoecd/60/31/2762731.pdf>. pp. 4. Acesso em 21/10/2009

OECD Directorate for Science, Technology and Industry: STI Outlook 2002: National Policies. REPUBLIC OF KOREA Disponível em: <http://www.oecd.org/dataoecd/59/47/2762668.pdf>. Acesso em 20/10/2009;

OECD Directorate for Science, Technology and Industry: STI Outlook 2004: National Policies. Republic of Korea Disponível em: <http://www.oecd.org/dataoecd/5/25/38893157.pdf>. Acesso em 20/10/2009.

OECD STI Outlook 2006 - Policy Questionnaire - Germany. Disponível em: <http://www.oecd.org/ dataoecd/5/2/38893058.pdf>. Acesso em 25/11/2009.

OECD. STI Outlook 2002 - Country Response to Policy Questionnaire - Republic of Korea

OECD. (2003b). Relatório Oficial sobre Governança corporativa na América Latina.

OECD. (2008). Science and Innovation: Country Notes - Germany. Disponível em: <http:// www.oecd.org/dataoecd/17/40/41559102.pdf>. Acesso em 25/10/2009.

OECD. Directorate for Science, Technology and Industry: STI Outlook. 2000: National Policies. Kore.2000. Disponível em: <http://www.oecd.org/dataoecd/25/48/2109999.pdf>. Acesso em 20/10/2009

OECD. Science, Technology and Industry Outlook 2004 - Country Response to Policy Questionnaire Republic of Korea. Disponível em <http://www.oecd.org/dataoecd/30/60/34242958.pdf>.

OECD. STI Outlook 2002 - Country Response to Policy Questionnaire - Germany. Disponível em: <http://www.oecd.org/dataoecd/60/1/2762626.pdf>. Acesso em: 25/10/2009; OECD (2004)

OECD. STI Outlook 2004 - Country Response to Policy Questionnaire - Germany. Disponível em: <http://www.oecd.org/dataoecd/30/42/34242808.pdf>. Acesso em 25/10/2009.

ORGANIZATION FOR ECONOMIC CO-OPERATION AND DEVELOPMENT - OECD. (2003 a) White Paper on Corporate Governance in Asia.

REPUBLIC OF KOREA - Ministry of Knowledge Economy. Pharmaceutical/BT 2008: Overview of Republic of Korea's Industries - Pharmaceutical/BT 2008 Invest REPUBLIC OF KOREA Published in October 2008. Disponível a partir de <http://www.mke.go.kr/language/eng/invest/ publications.jsp>. Acesso em 08 de dezembro de 2009.

REPUBLIC OF KOREA. Auto Parts 2008: Overview of Republic of Korea's Industries - Auto Parts 2008 Invest REPUBLIC OF KOREA, Published in October 2008". Disponível em http://www.mke.go.kr/ language/eng/invest/publications.jsp >. Acesso em 08 de dezembro de 2009.

REPUBLIC OF KOREA. Information and Communications 2008: Overview of Republic of Korea's Industries - Information and Communications 2008 Invest REPUBLIC OF KOREA Published in October 
2008 Disponível a partir de <http://www.mke.go.kr/language/eng/invest/publications.jsp >.Acesso em 08 de dezembro de 2009.

REPUBLIC OF KOREA. Ministry for Health, Welfare and Family Affairs. Outline of the heath care industries. Disponível em <http://english.mohw.go.kr/front_eng/jc/sjc0102mn.jsp?PAR_MENU_ID= 1003\&MENU_ID=100302>. Acesso em 08 de dezembro de 2009.

REPUBLIC OF KOREA. Ministry of Knowledge Economy Aerospace 2008: Overview of Republic of Korea's Industries - Aerospace 2008 Invest REPUBLIC OF KOREA

Published in October 2008. Disponível a partir de <http://www.mke.go.kr/language/eng/invest/ publications.jsp>. Acesso em 08 de dezembro de 2009.

REPUBLIC OF KOREA. Ministry of Land, Transport and Maritime Affairs. Ship Investment Company. Disponível em <http://english.mltm.go.kr/USR/WPGE0201/m_19527/DTL.jsp>. Acesso em 08 de dezembro de 2009.

REPUBLIC OF KOREA. Ministry of National Defense. Defense Plane - Outline. Disponível em <http:// www.mnd.mil.kr/mndEng/DefensePolicy/MasterPlan/overview/index.jsp>.

Acesso em 08 de dezembro de 2009

REPUBLIC OF KOREA. Vision 2030' calls for active overseas energy development. Disponível em < http://www.Republic of Korea.net/News/News/NewsView.asp?serial_no=20061130033>. Acesso em 08 de dezembro de 2009.

UNITED NATIONS. A visão de longo prazo para ciência e tecnologia para 2025. Disponível em <http://unpan1.un.org/intradoc/groups/public/documents/APCITY/UNPAN008049.pdf> Acesso em 20/11/2009.

USA (2004) Project BioShild . Disponível em <http://www.hhs.gov/aspr/barda/bioshield/ index.html>. Acesso em 08 de dezembro de 2009

USA. (2006) National Aeronautics Research and Development Policy. <http://www.ostp.gov/galleries/ Issues/National\%20Aero\%20Policy\%202006\%20(Glossy).pdf>. Acesso em 08 de dezembro de 2009

USA. "FutureGen Clean Coal Project", ver <http://www.fossil.energy.gov/programs/powersystems/ futuregen/ > . Acesso em 08 de dezembro de 2009.

USA. Advanced Energy Initiative. Disponível em <http://www.lgprogram.energy.gov/ energy_booklet.pdf>. Acesso em 08 de dezembro de 2009

USA. NITRD. (2007) Federal High End Computing Update High. Disponível em <http:// www.nitrd.gov/Subcommittee/hec/2005_sc_hec_bof.pdf.>. Acesso em 08 de dezembro de 2009

USA. NITRD. (2008) Interagency Working Group on High End Computing. Disponível em <http:// www.nitrd.gov/Subcommittee/hec.aspx>. Acesso em 08 de dezembro de 2009

USA. Office of Science and Technology Policy. Executive Office of the President. Hydrogen Fuel Initiative. Disponível em <http://www.ostp.gov/pdf/1pger_hydrogenfueliniative.pdf >. Acesso em 08 de dezembro de 2009

USA. Office of Science and Technology Policy. Executive Office of the President. Advanced Energy Initiative - Research and development in the President's 2009 Budget. Disponivel em <http:// www.ostp.gov/galleries/budget09/AdvancedEnergylnitiative1 pager.pdf $>$. Acesso em $08 \mathrm{de}$ dezembro de 2009

USA-NASA.(2004)President Bush Offers New Vision For NASA. Disponível em http://www.nasa.gov/ missions/solarsystem/bush_vision.html. . Acesso em 08 de dezembro de 2009.

USA-NIST. (2006) American Competitiveness Initiative Disponível em <http://www.nist.gov/director/ reports/ACIBooklet.pdf>. Acesso em 20/10/2009. 